\title{
Role of ion beam assisted deposition in the synthesis and fracture of metal-ceramic multilayers
}

\author{
G. S. Was \\ Department of Nuclear Engineering and Department of Materials Science and Engineering, 213 Cooley Building, University of Michigan, Ann Arbor, \\ MI 48109 (USA)
}

J. W. Jones

Department of Materials Science and Engineering, 2018 Dow Building. University of Michigan. Ann Arbor. MI 48109 (USA)

C. E. Kalnas and L. J. Parfitt

Department of Materials Science and Engineering, 124 NAME Building, University of Michigan, Ann Arbor, MI 48109 (USA)

M. Goldiner

Department of Nuclear Engineering, 232 Cooley Building, University of Michigan, Ann Arbor, MI 48109 (USA)

\begin{abstract}
Multilayer metal-ceramic films have the potential to serve as strong, tough and environmentally resistant films and coatings for a wide variety of applications. They derive their properties from the multilayer structure (architecture), the microstructure and, hence, mechanical properties of the individual layers and the stress state of the film. However, in order to realize the potential of microlaminates, these features (architecture, microstructure and stress) must be controlled. Ion beam assisted deposition (IBAD) holds the promise to provide this control. In an effort to understand how IBAD can control mechanical properties of films, single trilayer and five-bilayer metal-ceramic, $\mathrm{Al}-\mathrm{Al}_{2} \mathrm{O}_{3}$ films were fabricated on ductile metal substrates using IBAD over a range of thicknesses and normalized energies. Results of bending and tension experiments revealed that the stress state is critical in determining the fracture strain (ductility) of the film. A residual compressive stress is beneficial and can be formed in the oxide phase by bombardment of the film with Ar during deposition. The behavior of film stress correlates well with Ar gas incorporation and the film consists of a high density of small cavities. Gas incorporation into the cavities or the surrounding matrix may be responsible for the observed residual compressive stress. The density of surface cracks at high strains is a function of the film architecture, film strength and the interfacial shear strength. Use of a multilayer structure reduces the crack density over a monolithic oxide film by increasing the strain needed to form through-thickness cracks and by increasing the intrinsic strength of the brittle layers by decreasing their thickness. Ion bombardment of the metal layers resulted in radiation damage and grain size refinement, both of which result in a stronger film and a lower crack density. It was shown that architecture, microstructure and stress are the key ingredients in microlaminate properties and are uniquely controllable by IBAD.
\end{abstract}

\section{Introduction}

The development of microlaminate materials has received considerable interest in recent years because their mechanical properties are superior to those of bulk materials of similar composition. In particular, considerable activity, both theoretical $[1,2]$ and experimental [3-5], has been focused on the development of metal/ceramic and metal/intermetallic laminates which offer the potential for attractive combinations of strength and fracture toughness. It is the development of toughness or resistance to fracture which offers the greatest potential for the use of microlaminates as structural materials and coatings and it is in this area that microlaminate synthesis by ion assisted deposition, with its inherent ability to tailor properties of individual layers and interfaces, can be particularly important.
Numerous studies of deformation and fracture processes in metal/ceramic laminates have been performed in recent years and laminate architecture (e.g. ductile phase volume fraction and individual layer thicknesses), lamellae microstructure (grain size and morphology, texture, density) and mechanical properties, and stress state in the film have been identified as critical variables. Toughening in laminates results from energy dissipation by ductile fracture of the metal phase, by delamination of the metal-ceramic interface and by sliding or shearing of the interface [6]. Thus, modifications which increase the work of fracture of the ductile phase (increased thickness, increased tensile strength and greater failure strain) will improve microlaminate toughness. Microlaminate strength will depend on both metal and ceramic lamellae thickness and, of course, on the residual stress state. 
It is the control over each of these variables which is critical in achieving the optimum properties from microlaminate films. Architecture can be controlled by adjusting the duration of deposition, the deposition rate and by simultaneous or alternate use of multiple sources to control lamellae composition. Microstructure and stress state, however, are often impossible to control in conventional physical vapor deposition (PVD). This is where the utility of ion assisted deposition can make a large impact: by imparting control of the microstructure and stress state together with the architecture and, therefore, the mechanical properties of the film system.

In this paper we describe the fracture behavior of monolithic and multilayer films of $\mathrm{Al} / \mathrm{Al}_{2} \mathrm{O}_{3}$ in bending and tension. Residual stress behavior of the microlaminate and its components will be reviewed and a description of the microstructure will also be presented. The nature of the fracture process in microlaminates will be described in terms of its dependence on architecture, microstructure and stress state.

\section{Experimental details}

Ion beam assisted deposition (IBAD) was used to produce monolithic $\mathrm{Al}$ and $\mathrm{Al}_{2} \mathrm{O}_{3}$ films of varying thicknesses and $\mathrm{Al} / \mathrm{Al}_{2} \mathrm{O}_{3}$ microlaminates of either 3 or 10 layers. The deposition system consists of two $6 \mathrm{~kW}$ electron beam guns and a $3 \mathrm{~cm}$ Kaufman ion gun with dual thickness monitors and a Faraday cup for measuring the ion current. Deposition rates for each source were measured independently with quartz crystal monitors and the beam current was monitored with a Faraday cup. Results are reported in terms of either the ion-toatom arrival rate ratio $R$ or the normalized energy $E_{n}$, which is the product of the ion energy and $R: E_{\mathrm{n}}=R E$.

Monolithic films of $\mathrm{Al}$ and $\mathrm{Al}_{2} \mathrm{O}_{3}$ were deposited in thicknesses ranging from 400 to $1200 \mathrm{~nm}$ and from 150 to $2600 \mathrm{~nm}$ respectively. Microlaminates consisting of five $\mathrm{Al}_{2} \mathrm{O}_{3} / \mathrm{Al}$ bilayers (oxide on top) were made with two composition ratios, $45 \% \mathrm{Al}_{2} \mathrm{O}_{3} / 55 \% \mathrm{Al}$ and $80 \% \mathrm{Al}_{2} \mathrm{O}_{3} / 20 \% \mathrm{Al}$. Layer thicknesses were nominally $100 \mathrm{~nm}$ for the $45: 55$ microlaminate and $260 \mathrm{~nm}: 60 \mathrm{~nm}$ for the 80:20 microlaminate. Depositions were conducted at a base pressure below $4 \times 10^{-9}$ Torr for all films except 150 and $400 \mathrm{~nm}$ thick $\mathrm{Al}_{2} \mathrm{O}_{3}$ films which were deposited at a base pressure of $2 \times 10^{-7}$ Torr. Depositions were normal to the substrate at a rate of $0.7-2.0 \mathrm{~nm} \mathrm{~s}^{-1}$ for $\mathrm{Al}$ and $1.0 \mathrm{~nm} \mathrm{~s}^{-1}$ for $\mathrm{Al}_{2} \mathrm{O}_{3}$. Ion bombardment was performed using $200-700 \mathrm{eV} \mathrm{Ar}^{+}$ ions at an angle of $45^{\circ}$ to the substrate normal, with current densities in the range $1-190 \mu \mathrm{A} \mathrm{cm}^{-2}$, giving a range of $R$ ratios from 0.0025 to 0.5 . Trilayer films consisting of $300 \mathrm{~nm}$ layers of $\mathrm{Al}_{2} \mathrm{O}_{3} / \mathrm{Al}_{1} / \mathrm{Al}_{2} \mathrm{O}_{3}$ were deposited at the rate of $0.7-1.2 \mathrm{~nm} \mathrm{~s}^{-1}$ (oxide) and
$0.2-0.24 \mathrm{~nm} \mathrm{~s}^{-1}$ (metal) respectively under both PVD and IBAD (425-450 eV Ar ${ }^{+}$ions, $\left.R=0.01\right)$ conditions at a base pressure of $(1-4) \times 10^{-8}$ Torr. Depositions for bending and tensile experiments were made on 304 stainless steel (SS) and Al alloy 1100 substrates respectively of thickness $1 \mathrm{~mm}$ and width $4 \mathrm{~mm}$.

The amount and composition of films deposited onto graphite foil were determined by Rutherford backscattering analysis at an angle of $165^{\circ}$ and fitted with the aid of the program RUMP [7]. Thicknesses were measured using a Dektak model 2A profilometer. Grain size and morphology were determined from transmission electron microscopy (TEM) of planar and cross-section samples on a JEOL $2000 \mathrm{FX}$ TEMSCAN at $200 \mathrm{kV}$. Cavity size distribution in oxide films was determined using TEM and small angle $\mathrm{X}$-ray scattering using a Rigaku rotating anode X-ray spectrometer. Residual stress was determined by optical interferometry [8] on films and microlaminates deposited onto $150 \mu \mathrm{m}$ thick glass cover slips. The fracture behavior of $\mathrm{Al}_{2} \mathrm{O}_{3}$ films and $\mathrm{Al} / \mathrm{Al}_{2} \mathrm{O}_{3}$ microlaminates was investigated by monitoring the formation of film cracks during in situ bending of annealed 304 SS substrates with films from the first group of depositions (five bilayers) in an ElectroScan model E3 environmental scanning electron microscope and during tensile deformation of $1100 \mathrm{Al}$ substrates with films from the second group of depositions (trilayers).

\section{Results}

Ductile metal substrates, onto which selected monolithic and microlaminate films were deposited, were strained in either bending or tension to provide a measure of the relative fracture properties of the films. Figure 1 shows the development of the film crack density for PVD and $\operatorname{IBAD}(R=0.1)$ monolithic oxide films and five-bilayer $\mathrm{Al}_{2} \mathrm{O}_{3} / \mathrm{Al}$ microlaminates deposited on SS substrates subjected to bending. It should be noted that, for the monolithic oxide films, there is a large increase in the strain at which fracture starts to occur. There is also a decrease in the slope of the crack density vs. strain curve with ion bombardment or with the incorporation of $\mathrm{Al}$ metal layers. However, the critical strain for all the IBAD films is about the same. The saturation crack density is also a function of ion bombardment. In all cases (except for the PVD oxide which was not taken to saturation), IBAD films exhibit a lower saturation crack density.

Figure 2 shows results of tensile experiments for PVD oxide and both PVD and IBAD $(R=0.01)$ trilayer films on alloy $\mathrm{Al} 1100$. It should be noted that despite the difference in test type and substrate alloy, the general shape and trends of the data in Fig. 1 are reproduced in Fig. 2. Here again, the critical strain at fracture of the 


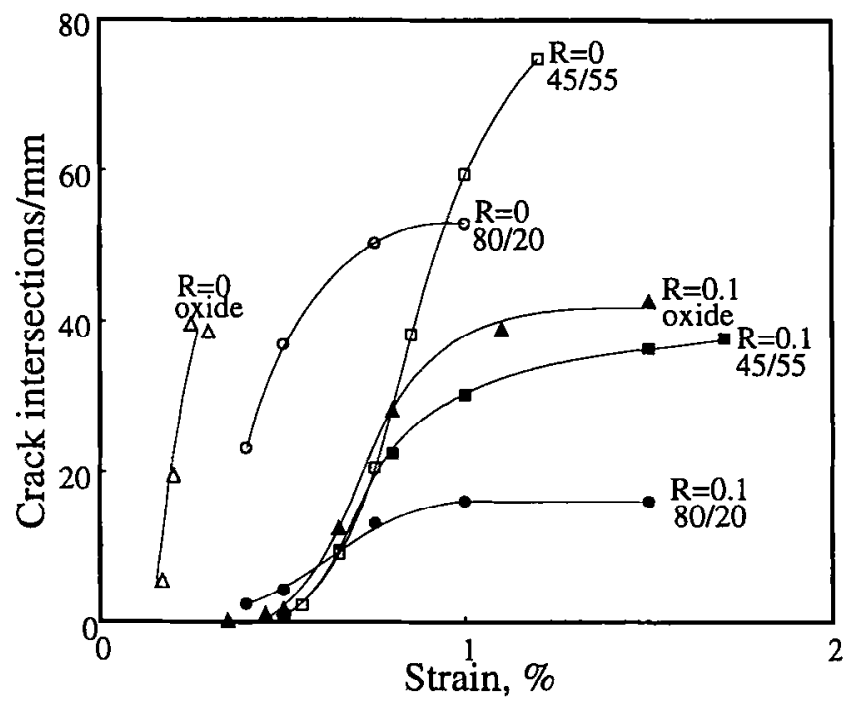

Fig. 1. Crack density $v s$. strain for oxide and five-bilayer microlaminate films on 304 SS substrates tested in bending.

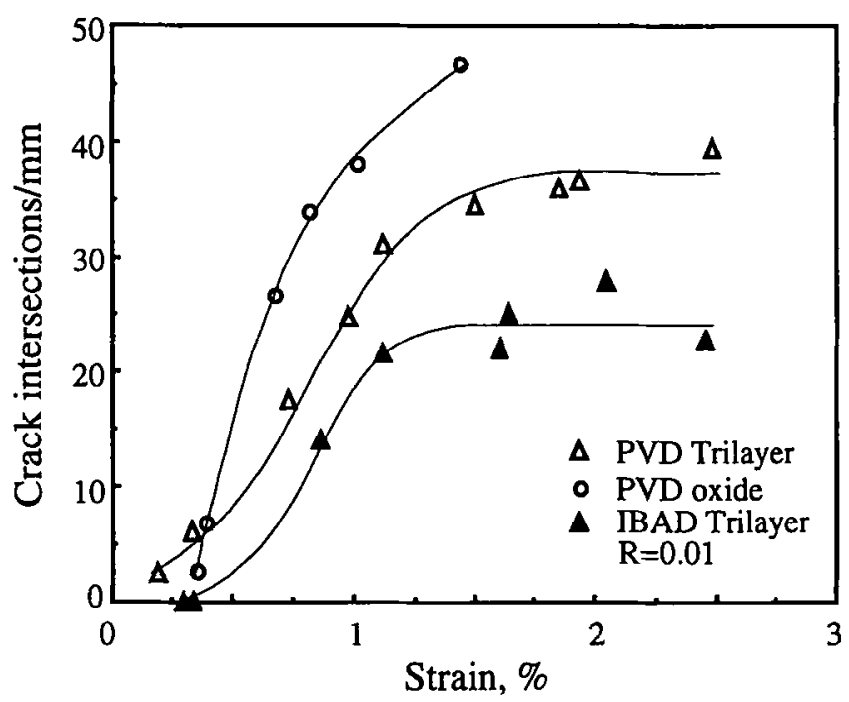

Fig. 2. Crack density us. strain for oxide and trilayer films on annealed aluminium alloy 1100 substrates subjected to tensile deformation.

oxide film is smaller and the slope is steeper than for either the PVD or the IBAD microlaminate. Also, the saturation crack density is highest for the PVD oxide, intermediate for the PVD microlaminate and lowest for the IBAD microlaminate.

Residual stresses in the monolithic oxide and metal films are shown in Fig. 3(a), gas incorporation in Fig. 3(b) and stress in the microlaminates in Fig. 4. It should be noted that the stress in the Al layer formed by PVD is slightly tensile and rises to a saturation value of about $0.13 \mathrm{GPa}$ with increasing normalized energy. The stress in the monolithic oxide layer varies in an inverse fashion from that of the metal layer. The relatively high tensile residual stresses produced by PVD are readily eliminated by ion bombardment and com-
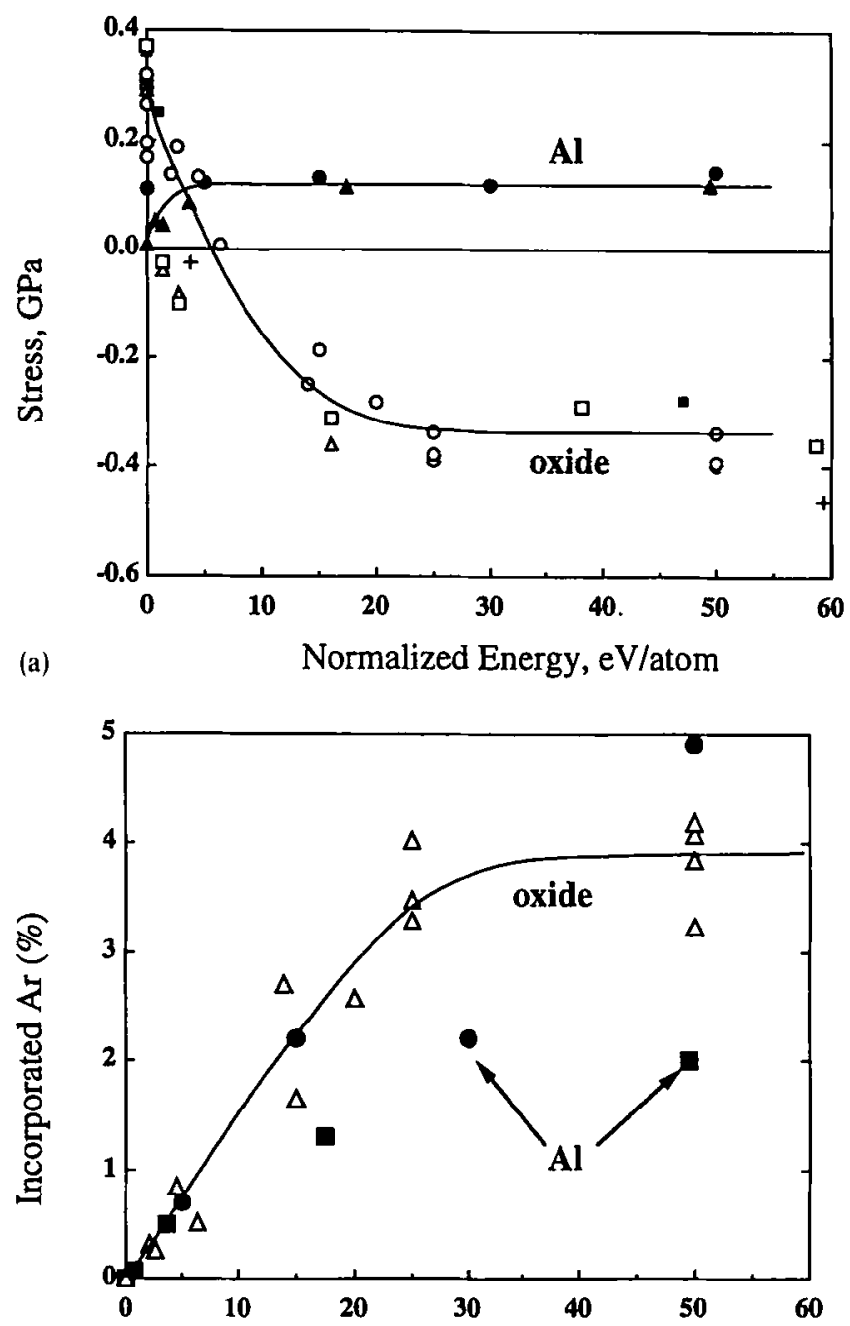

(b)

Normalized Energy, eV/atom

Fig. 3. (a) Residual stress in $400 \mathrm{~nm}$ and $1200 \mathrm{~nm}$ thick Al films (e, $\Delta)$ and $150-1650 \mathrm{~nm}$ thick $\mathrm{Al}_{2} \mathrm{O}_{3}$ films $(\square, \triangle, O)$ as a function of normalized energy, and (b) incorporated gas in $\mathrm{Al}$ and $\mathrm{Al}_{2} \mathrm{O}_{3}$ films as a function of normalized encrgy.

pressive stresses are produced at a very low normalized energy, $1.3 \mathrm{eV}$ atom $^{-1}(R=0.004)$. At values of $E_{\mathrm{n}}$ greater than about $15 \mathrm{eV}$ atom $^{-1}$ stresses saturate at about $-0.4 \mathrm{GPa}$. Residual stress appears to be relatively insensitive to film thickness. In both metal and oxide films, the Ar gas content rises to about $4 \%$ at $E_{\mathrm{n}}=50 \mathrm{eV}$ atom $^{-1}$.

The microstructure of the metal films made by PVD is characterized by large columnar grains $0.5 \mu \mathrm{m}$ in diameter extending through the thickness of the film. The PVD film has a strong, azimuthally symmetric (111) fiber texture. With increasing $E_{n}$, the grain size becomes refined, decreasing to $0.2 \mu \mathrm{m}$ at an $R$ ratio of 0.1 . The grain refinement is most pronounced at the substrate interface where a significantly higher nucleation density is observed. With increasing $E_{\mathrm{n}}$, the texture remains a (111) fiber texture, but a three-fold symmetry develops 


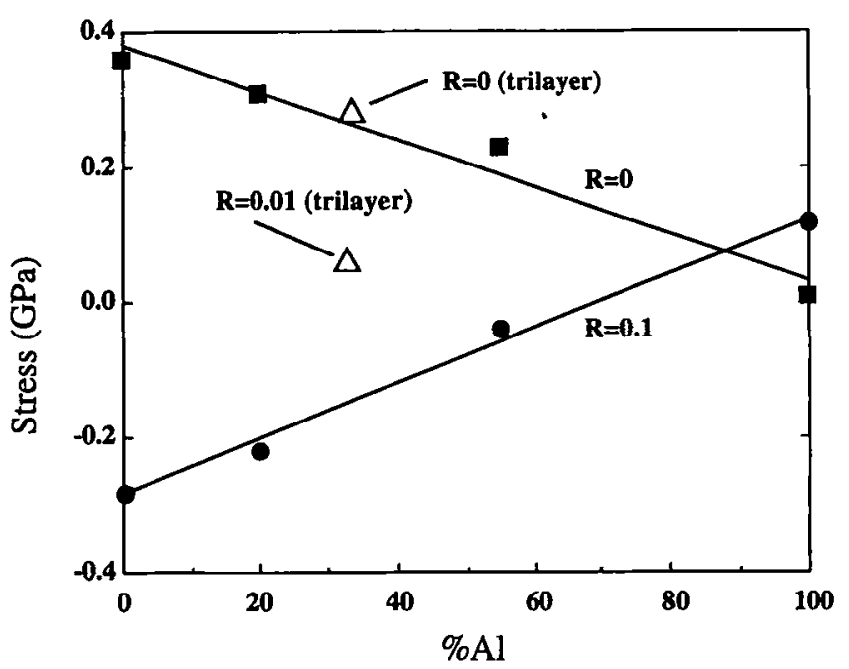

Fig. 4. Residual stress in five-bilayer $(\boldsymbol{\square}, \boldsymbol{\theta})$ and trilayer $(\Delta)$ microlaminate films as a function of aluminum content and deposition conditions.

which is consistent with planar channeling of the ion beam along (110). Ar is incorporated to a level of 4-5 at. $\%$ at the highest $R$ ratio of $0.1 \quad\left(E_{\mathrm{n}} \approx 50 \mathrm{eV}\right.$ atom $\left.^{-1}\right)$. The oxide layers are amorphous under all deposition conditions, are slightly substoichiometric and are quite porous, averaging about $70 \%$ theoretical density. The density does not change with increasing $R$ ratio or $E_{\mathrm{n}}$. The porosity is in the form of voids (or bubbles) in the $4 \mathrm{~nm}$ size range and with a density of $3 \times 10^{18} \mathrm{~cm}^{-3}$.

\section{Discussion}

The discussion will focus on two main points noted in the results of the fracture behavior of the films: (1) the increase in the fracture strain of the monolithic oxide with an ion assist (Fig. 1) and (2) the drop in the saturation crack density between oxide and microlaminate films and between PVD and IBAD microlaminates (Fig. 2). Discussion will draw on results of the stress analysis and microstructural characterization to provide a basis for understanding the fracture behavior as well as the observed behavior of film stress.

\subsection{Fracture strain}

During straining, cracks form perpendicular to the direction of maximum tensile stress. The strain at which the cracks begin to appear gives a measure of the tensile strength of the film. A plot of the crack density vs. the applied strain is sigmoidal and the fracture strain is taken to be the inflection point in the sigmoidal curve. The change in the fracture strain in the PVD and IBAD oxides shown in Fig. 1 can be understood in terms of residual stress in the deposited film. The film stress is a superposition of the applied stress and the residual stresses:

$\sigma_{\mathrm{t}}=\sigma_{\mathrm{a}}+\sigma_{\mathrm{r}}$

where $\sigma_{\mathrm{a}}$ and $\sigma_{\mathrm{r}}$ are applied and residual stresses respectively. Both PVD and IBAD films will start to crack when the stress in the film reaches the fracture stress. Since the PVD film is in tension while the IBAD film is in compression, the IBAD film will require a greater applied stress for the total stress to reach the fracture stress. From Fig. 3, the residual stresses in the PVD and IBAD films are $0.4 \mathrm{GPa}$ and $-0.4 \mathrm{GPa}$, respectively. Assuming an elastic modulus of $175 \mathrm{GPa}$ for amorphous alumina [9], the difference in applied strain needed to reach the fracture stress is about $0.46 \%$, which compares well with the value of about $0.55 \%$ strain from the measurements in Fig. 1. This analysis also qualitatively explains the smaller differences in fracture strain shown in Fig. 2.

\subsection{Crack density}

Above the critical strain for crack nucleation, crack density increases by the formation of new cracks and limited propagation of existing cracks. Eventually a saturation in crack density, defined by a maximum crack spacing $\lambda_{\max }$, will be achieved. Saturation arises when the separation between cracks is insufficient to allow tensile stresses in the film to exceed the critical fracture stress. This process is similar to the shear-lag model of stress transfer to the reinforcement phase in composites $[10]$ and has been quantified for the case of the fracture of brittle thin films on ductile substrates by Agrawal and $\operatorname{Raj}[11,12]$. Here the crack density (or maximum crack spacing) is related to the interfacial shear strength $\tau$ the tensile strength of the film $\sigma$, and the film thickness $\delta$, by

$\rho_{\mathrm{sat}}=1 / \lambda_{\max }=\tau / \pi \delta \sigma$

and would be expected to decrease with a decrease in the shear strength of the film-substrate interface, and with an increase in film strength for constant film thickness. Within this framework it is possible to describe, although qualitatively at this point, the differences in crack saturation densities between monolithic and multilayer films as well as further differences between PVD and IBAD multilayer films.

The saturation crack density in multilayers is lower than that observed in monolithic films of similar total thickness. The decrease in crack density at saturation in the microlaminate can be attributed to an increase in the difficulty of transmitting stresses from the deforming substrate to the film, which is a direct consequence of the presence of the aluminum layers and the extra $\mathrm{Al}-\mathrm{Al}_{2} \mathrm{O}_{3}$ interfaces. Interface delamination and plastic deformation of the aluminum layers have the effect of increasing the strain necessary to form through-thickness 
cracks in the multilayer film and this can, in effect, be described as a reduction in the effective shear strength of the substrate-film interface. Furthermore, it is necessary to incorporate the influence of the change in thickness of the brittle $\mathrm{Al}_{2} \mathrm{O}_{3}$ layers on film strength. The substitution of multilayers of $\mathrm{Al}_{2} \mathrm{O}_{3}$ separated by ductile $\mathrm{Al}$ layers, for an equivalent thickness of monolithic $\mathrm{Al}_{2} \mathrm{O}_{3}$, has the added effect of reducing the thickness of individual $\mathrm{Al}_{2} \mathrm{O}_{3}$ layers. Because the fracture strength of the brittle layers should vary inversely with thickness, their intrinsic strength should increase in the multilayers [6], promoting a further decrease in saturation crack density, as suggested in eqn. (2). Therefore, a decrease in the crack density can be due to either a reduction in the effective interfacial shear strength or an increase in the individual layer strength.

These same factors can also influence the slope of the crack density curve. Here again, a decrease in the efficiency of stress transfer from the substrate to the uncracked regions of the film and changes in the distribution of flaw sizes (strength) in the brittle layers will both lead to a decrease in the slope of the crack density curve with strain.

The effect on saturation crack density of ion bombardment during microlaminate deposition can also be rationalized in the context of eqn. (2). The efficiency of the aluminum layers in increasing film fracture resistance will increase as the flow strength and ductility of the aluminum increases [6], and, to a lesser extent, the $\mathrm{Al}-\mathrm{Al}_{2} \mathrm{O}_{3}$ interface strength. It is expected that the substantial grain refinement and the introduction of lattice defects resulting from the ion assist will substantially increase the work of rupture in the aluminum layers. This will, in effect, further increase the difficulty of propagating a crack from the substrate-film region to the film surface and have the net result of lowering the saturation crack density.

Evidence of the fracture processes and the strengthening of the aluminum layers described above can be seen in the scanning electron microscopy (SEM) fractographs of Figs. 5 and 6 . Figure 5 shows views normal to the plane of the film of a typical crack in an IBAD and in a PVD trilayer on an aluminum substrate subjected to tensile deformation. Film cracking in the PVD trilayer (Fig. 5(a)) is accompanied by apparent intergranular fracture of the aluminum interlayer and subsequent pullout of the film during fracture. However, this type of behavior is not observed in the IBAD trilayer, indicating that an improvement in both interface strength and film strength has occurred. This is further substantiated in Fig. 6, which shows the fracture appearance of similar IBAD and PVD trilayers that were deposited on silicon which was subsequently fractured to reveal the film cross-sections. There is a striking difference in the deformation of the Al layers in the direction normal to the

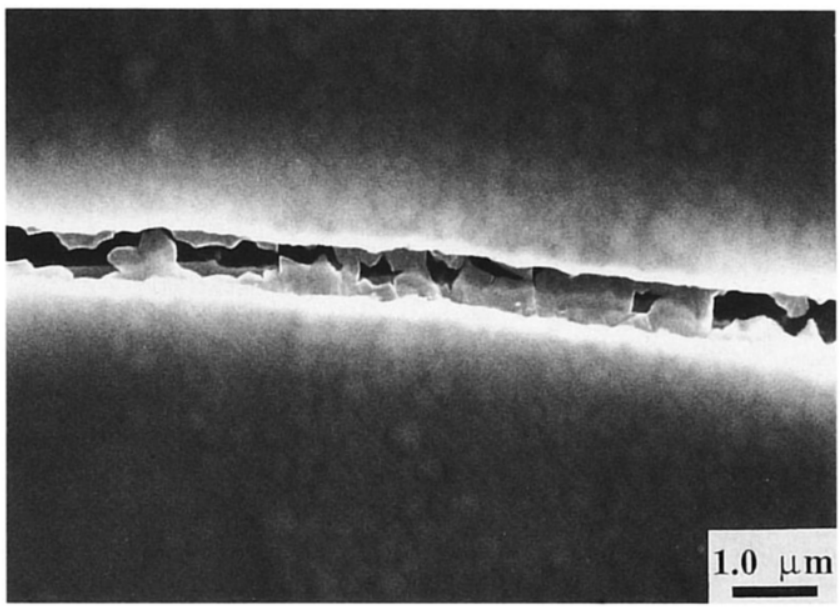

(a)

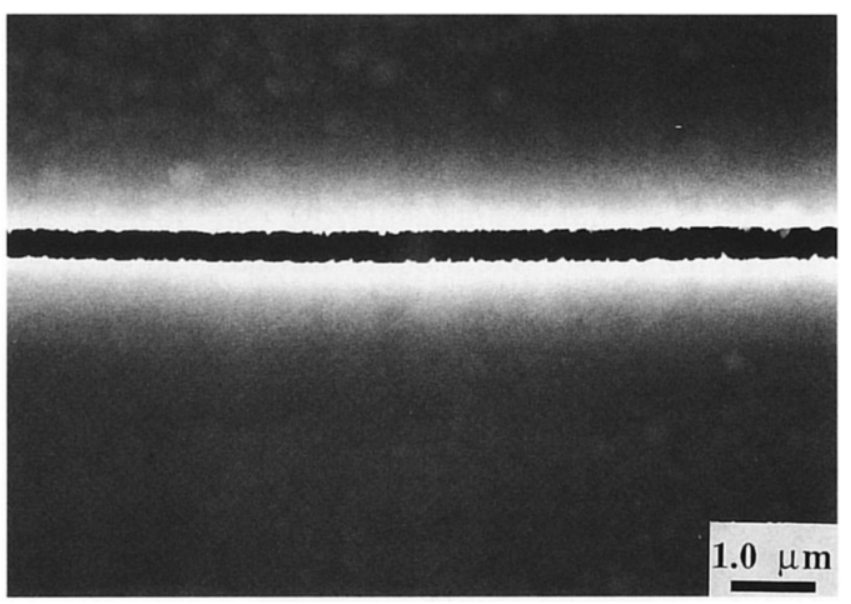

(b)

Fig. 5. SEM images of cracks in (a) PVD and (b) IBAD trilayers following tensile deformation.

film surface. While the PVD Al appears to undergo both grain deformation and intergranular detachment, neither occurs in the IBAD Al film. This is probably due to grain refinement which, in combination with irradiationinduced defects, strengthens the $\mathrm{Al}$ grains and grain boundaries. Interface delamination still occurs in IBAD, as well as PVD films, although it appears that the first $\mathrm{Al}-\mathrm{Al}_{2} \mathrm{O}_{3}$ interface is stronger in the IBAD film, perhaps because of the high density of grain nuclei from ion bombardment.

An explanation for the very low crack density in the 80:20 IBAD microlaminate in Fig. 1 is required. This is a special case, as can be seen from the micrograph in Fig. 7. The IBAD film undergoes buckling in the width direction of the sample while no buckling occurs in the PVD film. This results directly from the large compressive biaxial residual stress in this film. During deformation in the bend test, the fracturc stress normal to the maximum applied tensile stress is exceeded and, when parallel cracks form, the unrelieved in-plane compressive 


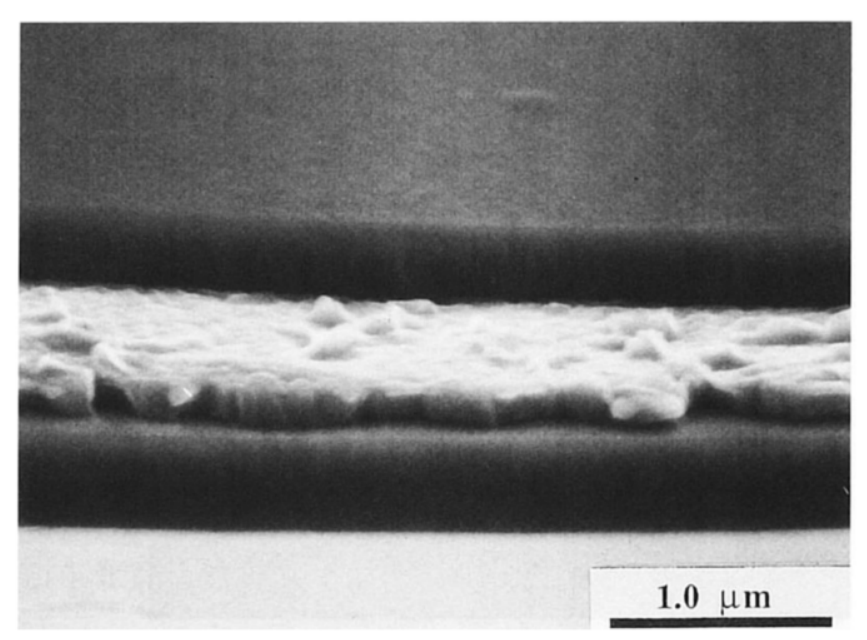

(a)

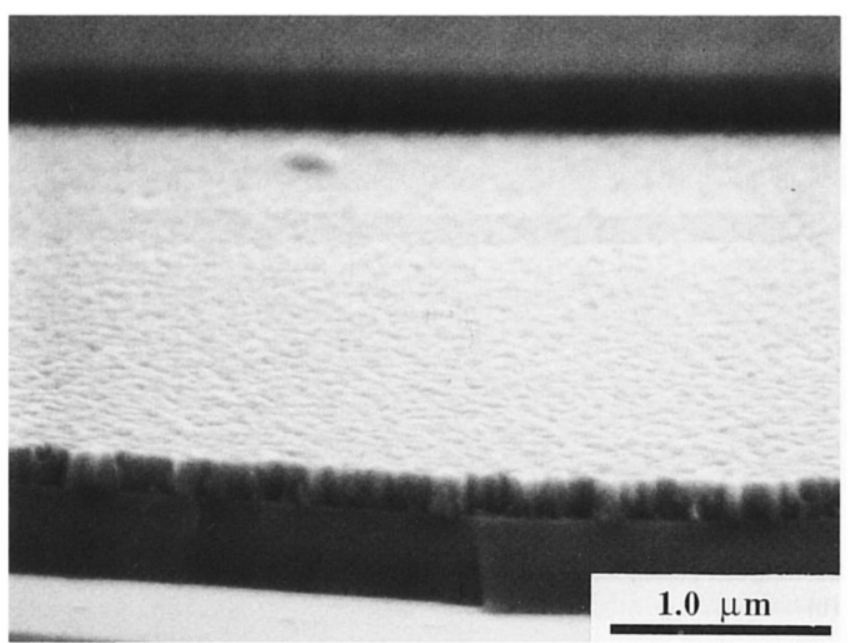

(b)

Fig. 6. Cross-sections of (a) PVD and (b) IBAD trilayers revealed by fracture of the silicon substrate and trilayers.

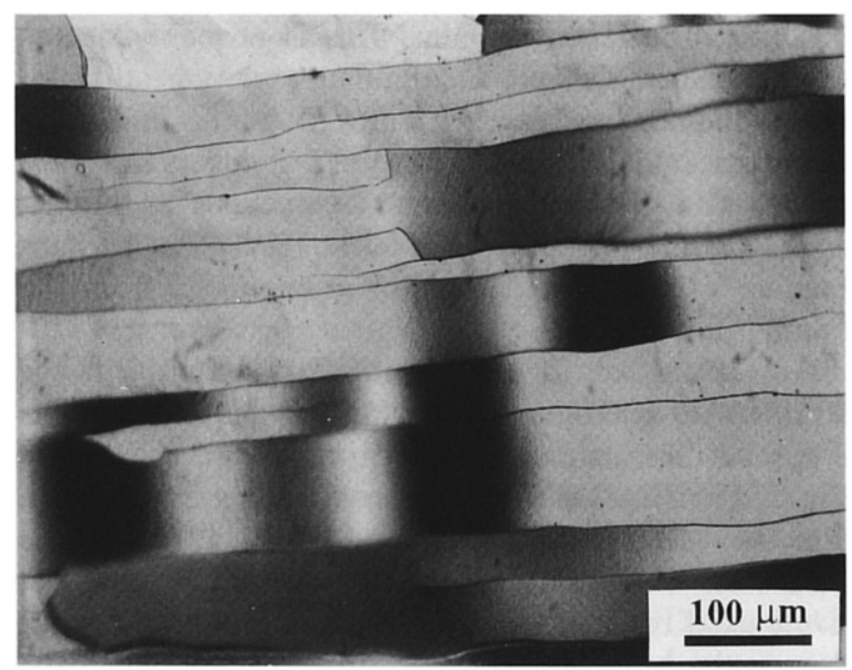

Fig. 7. Film cracks at saturation for a five-bilayer, $80: 20 \mathrm{Al}_{2} \mathrm{O}_{3}: \mathrm{Al}$ film $\left(E_{\mathrm{n}}=50 \mathrm{eV}\right.$ atom $\left.^{-1}\right)$ on a 304 SS substrate in bending. stress parallel to the crack direction causes buckling. The buckling results in delamination of the film from the substrate, preventing further cracking in the direction of maximum tensile stress, and gives rise to the lower crack density observed in Fig. 7.

\subsection{Residual stress}

Since stress has been shown to have such a profound effect on fracture behavior in films, an understanding of film stress control, and hence its origin, becomes imperative. The residual stress in the microlaminate films is shown in Fig. 4 where stress is plotted $v s$. percentage of Al for $R=0,0.01$ and 0.1 . It should be noted that, for all $R$ ratios, the stresses for the microlaminates fall between the magnitudes of the terminal points. Also it should be noted that, in the cases for $R=0$ and 0.1 , the stresses in the microlaminates appear to follow a rule of mixtures of the terminal phases. This is particularly significant since the sign of the stress in the oxide phase is reversed between $R=0$ and $R=0.1$ and the difference in magnitude is quite large. It is also interesting to note that the stress for the PVD trilayer agrees with that for the five-bilayer microlaminate, and the IBAD trilayer with $R=0.01$ falls between the $R=0$ and $R=0.1$ curves, as it should. Thus, stress in microlaminates strongly adheres to a superposition rule.

Deposition of Al metal produces a tensile residual stress which increases from nearly zero at $R=0$ to about $0.13 \mathrm{GPa}$ with increasing $E_{\mathrm{n}}$ (Fig. 3(a)), in agreement with measurements by Park and Danyluk [13] and Dietz et al. [14]. The sign of the stress (tensile) is believed to be governed principally by the thermal mismatch with the Si substrate. Film/substrate temperature during deposition is estimated to be between 150 and $200^{\circ} \mathrm{C}$ from grain size measurements made from the PVD sample [15] and results in tensile stresses of about 0.1 to $0.15 \mathrm{GPa}$ on cool-down, in agreement with measurements. The effect of thermal mismatch was tested with a deposition at $-100^{\circ} \mathrm{C}$, which, when corrected for thermal expansion, results in stresses which agree well with those deposited under ambient conditions. The low stress under PVD conditions is believed to be due to the inability of the soft $\mathrm{Al}$ film to support a stress. IBAD produces grain size refinement and lattice damage, both of which will increase the strength and allow the film to support a higher stress.

The behavior of film stress in the oxide is not so straightforward. The variation in average film stress in monolithic crystalline films under ion bombardment has been explained by Hirsch and Varga [16] and Davis [17] as being related to the number of atoms involved in the thermal spike which varies as $E^{-5 / 3}$, or ion peening which implies a stress which varies as $(m E)^{1 / 2}[18]$. These models are more applicable to fully dense polycrystalline films. In the case of films with significant 
porosity, gas incorporation may play a role. Figure 3(b) shows that there is indeed a strong correlation between the noble gas content and the residual stress as both increase in magnitude until about $15 \mathrm{eV}$ atom ${ }^{-1}$, at which point they saturate. It may be that the saturation of incorporated gas is responsible for the saturation in the residual stress. Incorporated gas can affect the residual stress state either through its presence in cavities or in the matrix. If gas is incorporated into cavities, the pressure will produce a compressive stress in the surrounding lattice. Incorporation of gas into matrix sites will also produce a compressive stress. The observation that the film density does not change with increasing $E_{\mathrm{n}}$ may be due to the stabilization of the void structure by gas incorporation, similar to the effect seen in sintering. However, insufficient evidence exists to verify this mechanism at this time.

\subsection{Microstructure}

Apart from architecture, microstructure controls all the properties of the microlaminate. The residual stress in both $\mathrm{Al}$ and oxide phases is due to changes in microstructure under ion bombardment. The strength and ductility of the Al layer as well are dependent on the microstructure. It was shown that grain refinement and hardening of the Al layers by ion bombardment causes the greatest effect on the saturation crack density in IBAD vs. PVD microlaminates. It is the latter effect which, next to residual stress control, may offer the greatest potential for the use of IBAD in improving microlaminate film toughness. The efficiency of the ductile layers in increasing toughness depends on their energy absorbing capability. This, in turn, is directly influenced by the plastic flow behavior, by the strength of the ductile-brittle layer interface and by the compliance of the ductile layer. Indeed, the elimination of the columnar grain structure through ion bombardment may result in substantial improvements in microlaminate toughening because of the simultaneous improvements in ductile layer strength and fracture strain when compared with that of PVD films.

\section{Summary}

The fracture behavior of microlaminates produced by IBAD is controlled by three features: (1) film architecture, (2) stress state and (3) microstructure. All the critical features of cracking can be explained by variations in these film features.

(1) The stress state in the microlaminate follows a superposition of stress states in the individual layers weighted by their volume fractions.

(2) The sign of the stress in the Al metal layers is controlled by the thermal stress and the hardness of the layer as determined by grain size and irradiation damage.

(3) The stress state in the oxide layer can be correlated with the incorporation of gas into the amorphous structure. Gas present as either bubbles or in the matrix as individual atoms or small atom clusters could account for the observed behavior.

(4) The microstructure of the Al film (grain size and hardness) is believed to affect the tensile strength of the microlaminate system and the saturation crack density of the microlaminate.

Thus, architecture, microstructure and stress state all combine to provide an explanation of the fracture behavior of monolithic and microlaminate films made by IBAD.

\section{Acknowledgments}

The authors acknowledge the Michigan Ion Beam Laboratory for Surface Modification and Analysis for the use of the IBAD facilities and the Electron Microscope Analysis Laboratory. The authors also acknowledge $\mathrm{Mr}$ Ali Mashayekhi for help with film dcposition and Dr. David Hoffman, Ford Research Laboratory, for assistance in residual stress measurement. This work was supported under NSF Grant DMR-9100361.

\section{References}

1 A. G. Evans and R. M. McMeekkng, Acta Metall., 34 (1986) 2435.

2 B. Budiansky, J. C. Amazigo and A. G. Evans, J. Mech. Phys. Solids, 36 (1988) 167

3 G. R. Odette, B. L. Chao, J. W. Sheckherd and G. E. Lucas, Acta Metall., 40 (1992) 2381.

4 H. C. Cao, B. J. Dalgleish, H. E. Deve, C. Elliott, A. G. Evans, R. Mehrabian and G. R. Odette, Acta Metall., 37 (1989) 2969.

5 L. S. Sigl, A. G. Evans, P. Mataga, R. M. McMeeking and B. J. Dalgleish, Acta Metall., 36 (1988) 946.

6 M. Y. He, F. E. Heredia, D. J. Wissuchek, M. C. Shaw and A. G. Evans, Acta Metall. Mater., 41 (1993) 1223.

7 L. R. Dolittle, Nucl. Instrum. Methods B, 9 (1985) 334.

8 J. A. Thornton, J. Tabock and D. W. Hoffman, Thin Solid Films, 64 (1979) 111.

9 C. J. McHargue, Defect Diffus. Forum, 57-58 (1988) 359.

10 S. R. Swanson, J. Eng. Mater. Technol., 111 (1989) 145.

11 D. C. Agrawal and R. Raj, Acta Metall., 37 (1989) 1265.

12 D. C. Agrawal and R. Raj, Mater. Sci. Eng., A126 (1990) 125.

13 H.-W. Park and S. Danyluk, in A. T. Barfknecht, J. P. Partridge, C. J. Chen and C.-Y. Li (eds.), Advanced Electronic Packaging Materials, Vol. 167, Materials Research Society, Pittsburgh, PA, 1990 , p. 365.

14 V. Dietz, P. Ehrhart, D. Guggi, G.-B. Haubold, W. Jäger, M. Prieler and W. Schilling, Nucl. Instrum. Methods B, 59-69 (1991) 284.

15 C. R. M. Grovenor, H. T. G. Hentzell and D. A. Smith, Acta Metall., 32 (1984) 773.

16 E. H. Hirsch and I. K. Varga, Thin Solid Films, 69 (1980) 99.

17 C. A. Davis, Thin Solid Films, 226 (1993) 30.

18 H. Windishman, Crit. Rev. Solid State Mater. Sci., 17 (6) (1992) 547. 